\title{
String- and Brane-Localized Causal Fields in a Strongly Nonlocal Model
}

\author{
Detlev Buchholz ${ }^{a}$ and Stephen J. Summers ${ }^{b}$ \\ ${ }^{a}$ Institut für Theoretische Physik, Universität Göttingen, \\ 37077 Göttingen, Germany \\ ${ }^{b}$ Department of Mathematics, University of Florida, \\ Gainesville FL 32611, USA
}

\begin{abstract}
We study a weakly local, but nonlocal model in spacetime dimension $d \geq 2$ and prove that it is maximally nonlocal in a certain specific quantitative sense. Nevertheless, depending on the number of dimensions $d$, it has string-localized or brane-localized operators which commute at spatial distances. In two spacetime dimensions, the model even comprises a covariant and local subnet of operators localized in bounded subsets of Minkowski space which has a nontrivial scattering matrix. The model thus exemplifies the algebraic construction of local operators from algebras associated with nonlocal fields.
\end{abstract}

\section{Introduction}

Until recently, locality has been viewed as a basic feature of quantum field theory. However, nonlocal theories arising naturally in the context of quantum gravity and string theory [16], in curved and noncommutative space-times $[11,13,15]$, and in recent approaches to the construction of local theories [10,11,19-22,26,27], throw a new light on this matter. In this paper we examine a prototype of a nonlocal theory in $d \geq 2$ spacetime dimensions from the point of view of these and other recent developments. The model is based on a weakly local but nonlocal quantum field, which satisfies all remaining Wightman axioms and the superstability conditions studied in [11].

In particular, we are interested in investigating just how nonlocal this model is and to which extent there are remnants of locality which have physical significance. We shall show that the model is maximally nonlocal in a specific, quantitative sense. Nonetheless, it contains "string-localized" or - depending on the number of spacetime dimensions - "brane-localized" operators which commute at spatial 
distances. In two spacetime dimensions, it contains a local, covariant net for which a full scattering theory can be defined, yielding a scattering matrix not equal to the identity. This illustrates in another concrete model a recently emerged approach $[10,11,19-22,26,27]$ to establish the existence of local theories by starting from algebras generated by nonlocal operators. The essential advantage of this novel approach is that the intricate explicit construction of local operators can be avoided by using algebraic techniques.

After introducing the model in the next section, we prove in Section 3 that it is maximally nonlocal. In Section 4 we compute its modular structure and establish some immediate consequences. We then investigate the independence properties of spacelike separated algebras in Section 5. In Section 6 we show that, depending on the number of spacetime dimensions, the nonlocal wedge algebras generated by the field contain point-, string- respectively brane-localized operators which satisfy the condition of locality. In the final section, we discuss the significance of these findings from a number of vantage points and make some further comments.

\section{The model}

The model we are studying in this paper has been known at least since R. Jost's classic monograph on axiomatic quantum field theory [17]. It describes a scalar massive Fermion and was used to establish that weak local commutativity of field operators is a strictly weaker condition than local commutativity in the context of Wightman's axioms.

We shall consider this model in Minkowski space-time of any dimension $d \geq 2$. It is defined on the antisymmetric Fock space $\mathcal{H}$ over the one-particle space $\mathcal{H}_{1}$ of a scalar particle of mass $m>0$, i.e. the direct sum of the antisymmetrized $n$-fold tensor products $\mathcal{H}_{n}=\mathcal{H}_{1} \wedge \cdots \wedge \mathcal{H}_{1}, n \in \mathbb{N}$, and the space $\mathcal{H}_{0}$ consisting of multiples of the vacuum state $\Omega$. We identify $\mathcal{H}_{1}$ with the $L^{2}$-space of momentum-space wave functions equipped with the standard Poincaré invariant scalar product and consider the linear mapping of the space of test functions $\mathcal{S}\left(\mathbb{R}^{d}\right)$ into $\mathcal{H}_{1}$ given by restricting the Fourier transforms $\tilde{f}$ of $f \in \mathcal{S}\left(\mathbb{R}^{d}\right)$ to the positive mass shell $M$

$$
|f\rangle \doteq \widetilde{f}\left\lceil\left\{p \in \mathbb{R}^{d}: p_{0}=\sqrt{\boldsymbol{p}^{2}+m^{2}}\right\} .\right.
$$

The scalar product of $|f\rangle,|g\rangle \in \mathcal{H}_{1}$ is given by

$$
\langle f \mid g\rangle=2 \pi \int d p \theta\left(p_{0}\right) \delta\left(p^{2}-m^{2}\right) \overline{\widetilde{f}(p)} \widetilde{g}(p) .
$$

The natural action of the Poincaré transformations $\lambda \in \mathcal{P}_{+}^{\uparrow}$ on the test functions, $(\lambda f)(x) \doteq f\left(\lambda^{-1} x\right), x \in \mathbb{R}^{d}$, induces a continuous unitary representation $U$ of $\mathcal{P}_{+}^{\uparrow}$ on $\mathcal{H}$ which leaves $\Omega$ invariant and acts on the generating vectors according to

$$
U(\lambda)\left|f_{1}\right\rangle \wedge \cdots \wedge\left|f_{n}\right\rangle=\left|\lambda f_{1}\right\rangle \wedge \cdots \wedge\left|\lambda f_{n}\right\rangle \text {. }
$$


The representation $U$ satisfies the relativistic spectrum condition (positivity of the energy in all Lorentz frames).

On the antisymmetric Fock space $\mathcal{H}$ one can introduce in a standard manner a field $\phi$ satisfying canonical anticommutation relations. Concretely, $\phi$ is the operator-valued distribution

$$
\phi: \mathcal{S}\left(\mathbb{R}^{d}\right) \longrightarrow \mathcal{B}(\mathcal{H})
$$

which acts on the generating vectors according to

$$
\begin{aligned}
& \phi(f)\left|f_{1}\right\rangle \wedge \cdots \wedge\left|f_{n}\right\rangle \\
& =|f\rangle \wedge\left|f_{1}\right\rangle \wedge \cdots \wedge\left|f_{n}\right\rangle+\sum_{k=1}^{n}(-1)^{k+1}\left\langle\bar{f} \mid f_{k}\right\rangle\left|f_{1}\right\rangle \wedge \cdots \vee \cdots \wedge \wedge\left|f_{n}\right\rangle,
\end{aligned}
$$

where $\stackrel{k}{\vee}$ denotes omission of $\left|f_{k}\right\rangle$. It follows from this definition that $\phi$ satisfies the Klein-Gordon equation

$$
\phi\left(\left(\square+m^{2}\right) f\right)=0
$$

the anticommutation relations

$$
\{\phi(f), \phi(g)\} \doteq \phi(f) \phi(g)+\phi(g) \phi(f)=(\langle\bar{g} \mid f\rangle+\langle\bar{f} \mid g\rangle) \cdot 1
$$

and the hermiticity condition

$$
\phi(f)^{*}=\phi(\bar{f})
$$

Moreover, the unitary representation $U$ of the Poincaré group acts covariantly on the field,

$$
U(\lambda) \phi(f) U(\lambda)^{-1}=\phi(\lambda f)
$$

Thus $\phi$ is a scalar Fermi field which, in accordance with the Spin-StatisticsTheorem, is nonlocal.

As is well known, one can decompose the field $\phi$ into creation and annihilation parts, $\phi(f)=\phi_{+}(f)+\phi_{-}(f)$, whose respective actions on the generating vectors are given by the first and second term on the right hand side of equation (2.1). So one obtains further anticommutation relations

$$
\left\{\phi_{+}(f), \phi(g)\right\}=\langle\bar{g} \mid f\rangle \cdot 1, \quad\left\{\phi_{-}(f), \phi(g)\right\}=\langle\bar{f} \mid g\rangle \cdot 1 .
$$

For our purposes here, we must introduce some further objects. Setting

$$
V=(-1)^{N(N-1) / 2}, \quad Z=(-1)^{N},
$$

where $N$ is the particle number operator acting on the subspaces $\mathcal{H}_{n} \subset \mathcal{H}$ by multiplication with $n, n \in \mathbb{N}_{0}$, we define an additional field $\widehat{\phi}: \mathcal{S}\left(\mathbb{R}^{d}\right) \rightarrow \mathcal{B}(\mathcal{H})$. It is given by

$$
\widehat{\phi}(f) \doteq V \phi(f) V^{-1}=\left(\phi_{+}(f)-\phi_{-}(f)\right) Z,
$$


where the latter equality follows from the fact that $\phi_{ \pm}(f)$ changes the particle number by \pm 1 and $(-1)^{N^{2}}=Z$. Since $V$ commutes with all Poincaré transformations $U(\lambda)$, the field $\widehat{\phi}$ transforms in the same covariant manner under the adjoint action of these transformations as the original field $\phi$.

Although the fields $\phi, \widehat{\phi}$ are nonlocal, they are relatively local, as is seen by the following computation. Noticing that $Z$ and $\phi$ anticommute, $Z \phi(g)+\phi(g) Z=0$, one obtains for the commutator

$$
[\widehat{\phi}(f), \phi(g)]=-\left\{\phi_{+}(f)-\phi_{-}(f), \phi(g)\right\} Z=(\langle\bar{f} \mid g\rangle-\langle\bar{g} \mid f\rangle) Z .
$$

But the expression

$$
\langle\bar{f} \mid g\rangle-\langle\bar{g} \mid f\rangle=2 \pi \int d p \varepsilon\left(p_{0}\right) \delta\left(p^{2}-m^{2}\right) \widetilde{f}(-p) \widetilde{g}(p)
$$

is the smeared commutator distribution affiliated with the Klein-Gordon equation, cf. [17], which vanishes if the supports of the test functions $f$ and $g$ are spacelike separated.

We consider in the following von Neumann algebras which are generated by the fields smeared with test functions having support in given subregions of Minkowski space. First, for any nonempty open subset $\mathcal{O} \subset \mathbb{R}^{d}$, we define $\mathcal{R}(\mathcal{O})$ to be the von Neumann algebra generated by the set of operators

$$
\left\{\phi(f): f \in \mathcal{S}\left(\mathbb{R}^{d}\right), \operatorname{supp} f \subset \mathcal{O}\right\} .
$$

With this definition, the map $\mathcal{R}: \mathcal{O} \mapsto \mathcal{R}(\mathcal{O})$ is clearly inclusion preserving and thus defines a net on $\mathbb{R}^{d}$. Due to the covariance properties of the field $\phi$, this net transforms covariantly under Poincaré transformations, i.e.

$$
U(\lambda) \mathcal{R}(\mathcal{O}) U(\lambda)^{-1}=\mathcal{R}(\lambda \mathcal{O})
$$

but it is not local, i.e. it is not true that the elements of the algebras $\mathcal{R}\left(\mathcal{O}_{1}\right)$ and $\mathcal{R}\left(\mathcal{O}_{2}\right)$ commute whenever the regions $\mathcal{O}_{1}$ and $\mathcal{O}_{2}$ are spacelike separated.

The second net $\widehat{\mathcal{R}}: \mathcal{O} \mapsto \widehat{\mathcal{R}}(\mathcal{O})$ is generated in the same way by the field $\widehat{\phi}$. Thus, $\widehat{\mathcal{R}}(\mathcal{O})=V \mathcal{R}(\mathcal{O}) V^{-1}$ for every $\mathcal{O} \subset \mathbb{R}^{d}$, so this net is Poincaré covariant and nonlocal as well. Yet, because of the relative locality of the fields $\phi$ and $\widehat{\phi}$ established above, the elements of the algebras $\mathcal{R}\left(\mathcal{O}_{1}\right)$ and $\widehat{\mathcal{R}}\left(\mathcal{O}_{2}\right)$ do commute whenever $\mathcal{O}_{1}$ and $\mathcal{O}_{2}$ are spacelike separated. Denoting the spacelike complement of $\mathcal{O}$ by $\mathcal{O}^{\prime}$ and the commutant of $\mathcal{R}(\mathcal{O})$ by $\mathcal{R}(\mathcal{O})^{\prime}$, we therefore have the following algebraic starting point.

Lemma 2.1 The two nets $\mathcal{R}, \widehat{\mathcal{R}}$ are $U\left(\mathcal{P}_{+}^{\uparrow}\right)$-covariant, nonlocal nets which are relatively local in the sense that one has

$$
\mathcal{R}(\mathcal{O}) \subset \widehat{\mathcal{R}}\left(\mathcal{O}^{\prime}\right)^{\prime}=V \mathcal{R}\left(\mathcal{O}^{\prime}\right)^{\prime} V,
$$

for any open $\mathcal{O} \subset \mathbb{R}^{d}$. 
In this paper a distinguished class of spacetime regions called wedges plays a special role. Choosing proper coordinates, let $W_{R} \doteq\left\{x \in \mathbb{R}^{d}\left|x_{1}>\right| x_{0} \mid\right\}$ denote the "right wedge" and $W_{L} \doteq\left\{x \in \mathbb{R}^{d}\left|-x_{1}>\right| x_{0} \mid\right\}=W_{R}^{\prime}$ the corresponding "left wedge". For $d>2$, the set $\mathcal{W}$ of wedges is given by $\mathcal{W}=\left\{\lambda W_{R} \mid \lambda \in \mathcal{P}_{+}^{\uparrow}\right\}$. In two dimensions, the set $\mathcal{W}$ of wedges has two disconnected components, one consisting of the translates of $W_{R}$ and the other of the translates of $W_{L}$.

\section{Degree of nonlocality of the model}

In order to gauge the nonlocality of the model under investigation, we employ a quantitative measure of commensurability of observables recently introduced in [12]. Let $\mathcal{A}, \mathcal{B}$ be two von Neumann algebras acting on some Hilbert space $\mathcal{H}$, and let $\mathcal{A} \bigvee \mathcal{B}$ be the von Neumann algebra generated by them. A measure of the strength of correlations between $\mathcal{A}$ and $\mathcal{B}$ in a normal state $\omega$ on $\mathcal{A} \vee \mathcal{B}$ is given by

$$
C_{\omega}(\mathcal{A}, \mathcal{B}) \doteq \sup _{E, F}|\omega(E \wedge F)-\omega(E) \omega(F)|
$$

where the supremum extends over all pairs of projections $E \in \mathcal{A}, F \in \mathcal{B}$, and $E \wedge F$ is the maximal projection contained in both $E$ and $F$. From this one can proceed to a measure of the incommensurability of the two algebras, setting

$$
C(\mathcal{A}, \mathcal{B}) \doteq \inf _{\omega} C_{\omega}(\mathcal{A}, \mathcal{B})
$$

the infimum being taken over all normal states on $\mathcal{A} \bigvee \mathcal{B}$

The values of $C(\mathcal{A}, \mathcal{B})$ can be shown to lie in the interval $[0,1]$, where the extreme value 0 implies the commutativity of $\mathcal{A}$ and $\mathcal{B}$, provided the algebra $\mathcal{A} \bigvee \mathcal{B}$ is simple, cf. [12]. The value 1 indicates that the two algebras are maximally incommensurable; it is realized, for example, in quantum mechanics by the algebras generated by the position and momentum operator, respectively. Thus, if for spacelike separated regions $\mathcal{O}_{1}, \mathcal{O}_{2}$ one has $C\left(\mathcal{R}\left(\mathcal{O}_{1}\right), \mathcal{R}\left(\mathcal{O}_{2}\right)\right)=1$, it is meaningful to say that the underlying net $\mathcal{R}$ is maximally nonlocal. We shall compute this invariant and show that the net in the present model is indeed maximally nonlocal. As a matter of fact, this result holds also for the subnet $\mathcal{R}_{e}$ generated by the even polynomials in the field $\phi$.

Let $f_{1}, f_{2}$ be real test functions which are orthonormal in the sense

$$
\left\langle f_{1} \mid f_{1}\right\rangle=\left\langle f_{2} \mid f_{2}\right\rangle=1, \operatorname{Re}\left\langle f_{1} \mid f_{2}\right\rangle=0
$$

so that $\phi\left(f_{1}\right), \phi\left(f_{2}\right)$ are self-adjoint involutions and $\phi\left(f_{1}\right) \phi\left(f_{2}\right)=-\phi\left(f_{2}\right) \phi\left(f_{1}\right)$. For such test functions, the operator $i \phi\left(f_{1}\right) \phi\left(f_{2}\right)$ is self-adjoint and unitary; hence it is an involution. It follows that $P_{ \pm}=\frac{1}{2}\left(1 \pm i \phi\left(f_{1}\right) \phi\left(f_{2}\right)\right)$ are orthogonal projections in $\mathcal{H}$ and $P_{+}+P_{-}=1$. Similarly, let $g_{1}, g_{2}$ be another pair of such test functions and set $Q_{ \pm}=\frac{1}{2}\left(1 \pm i \phi\left(g_{1}\right) \phi\left(g_{2}\right)\right)$. We assume that the supports of $f_{1}, f_{2}$ are spacelike separated from those of $g_{1}, g_{2}$. 
Lemma 3.1 Let $f_{1}, f_{2}$ and $g_{1}, g_{2}$ be pairs of test functions as described above such that $\left\langle f_{1}+i f_{2} \mid g_{1}-i g_{2}\right\rangle \neq 0$. Then one has $P_{+} \wedge Q_{+}=0$.

Proof. Setting $S=\frac{1}{2} \phi\left(f_{1}-i f_{2}\right), T=\frac{1}{2} \phi\left(g_{1}-i g_{2}\right)$, it is straightforward to verify the equalities

$$
\begin{aligned}
& S S^{*}=P_{+}, S^{*} S=P_{-}, T T^{*}=Q_{+}, T^{*} T=Q_{-}, \\
& S T+T S=\frac{1}{2}\left\langle f_{1}+i f_{2} \mid g_{1}-i g_{2}\right\rangle \cdot 1 .
\end{aligned}
$$

In the latter equality the fact was used again that $\langle\bar{f} \mid g\rangle=\langle\bar{g} \mid f\rangle$, if $f$ and $g$ have spacelike separated supports. Since $S=P_{+} S=S P_{-}$and $P_{+}+P_{-}=1$, one has $S P_{+}=S\left(1-P_{-}\right)=0$ and, similarly, $T Q_{+}=0$. Thus if $\Phi \in P_{+} \mathcal{H} \cap Q_{+} \mathcal{H}$, it follows that $S \Phi=T \Phi=0$ and consequently

$$
\frac{1}{2}\left\langle f_{1}+i f_{2} \mid g_{1}-i g_{2}\right\rangle \Phi=(S T+T S) \Phi=0 \text {. }
$$

Hence, under the given conditions on the test functions, $P_{+} \mathcal{H} \cap Q_{+} \mathcal{H}=\{0\}$, so that $P_{+} \wedge Q_{+}=0$.

Next, we construct sequences of functions with the properties specified above, which will be used to exhibit certain specific central sequences of projections.

Lemma 3.2 There exists a sequence $\left\{f_{1, n}, f_{2, n}\right\}_{n \in \mathbb{N}}$ of orthonormal pairs of real test functions, such that supp $f_{j, n} \subset\left\{x \in \mathbb{R}^{d}:|x|<1 / n\right\}, j=1,2$, and $\lim _{n \rightarrow \infty}\left\langle f_{1, n} \mid f_{2, n}\right\rangle=-i$. Moreover, $\lim _{n \rightarrow \infty}\left\langle f_{j, n} \mid g\right\rangle=0, j=1,2$, for any test function $g$.

Proof. Recall that the entire analytic "sine integral" Si is given by

$$
z \mapsto \operatorname{Si}(z)=\frac{2}{\pi} \int_{0}^{z} d w \frac{\sin w}{w},
$$

where the normalization is chosen in such a way that $\lim _{z \rightarrow \pm \infty} \operatorname{Si}(z)= \pm 1$; its Fourier transform is a tempered distribution with support in the interval $[-1,1]$. Let $x \mapsto h(x)$ be any real test function on $\mathbb{R}^{d}$ which is symmetric about the origin and has support in the ball $\left\{x \in \mathbb{R}^{d}:|x|<1 / 2\right\}$, and let $\widetilde{h}(0)=0$. Consider corresponding sequences of test functions $h_{1, n}, h_{2, n}, n \in \mathbb{N}$, given in momentum space by

$$
\begin{aligned}
& \widetilde{h}_{1, n}(p) \doteq\left(i / n^{d-2}\right) \operatorname{Si}\left(p_{0} / 2 n\right) \widetilde{h}\left(p / n^{2}\right) \\
& \widetilde{h}_{2, n}(p) \doteq\left(1 / n^{d-2}\right) \widetilde{h}\left(p / n^{2}\right)
\end{aligned}
$$

Upon multiplication with suitable normalization factors which will be determined below, these functions have all properties stated in the lemma. To verify this, begin by noting that the test functions $h_{1, n}, h_{2, n}$ are real. Moreover, in view of the 
support properties of $h$ and those of the Fourier transform of Si, the supports of $h_{1, n}$ and $h_{2, n}$ are contained in the ball $\left\{x \in \mathbb{R}^{d}:|x|<1 / n\right\}$. After a change of variables, one obtains for the scalar products

$$
\begin{aligned}
& \left\langle h_{1, n} \mid h_{1, n}\right\rangle=2 \pi \int d p \theta\left(p_{0}\right) \delta\left(p^{2}-m^{2} / n^{4}\right)\left|\operatorname{Si}\left(n p_{0} / 2\right)\right|^{2}|\widetilde{h}(p)|^{2}, \\
& \left\langle h_{2, n} \mid h_{2, n}\right\rangle=2 \pi \int d p \theta\left(p_{0}\right) \delta\left(p^{2}-m^{2} / n^{4}\right)|\widetilde{h}(p)|^{2}, \\
& \left\langle h_{1, n} \mid h_{2, n}\right\rangle=-2 \pi i \int d p \theta\left(p_{0}\right) \delta\left(p^{2}-m^{2} / n^{4}\right) \operatorname{Si}\left(n p_{0} / 2\right)|\widetilde{h}(p)|^{2} .
\end{aligned}
$$

The latter relation implies that $\operatorname{Re}\left\langle h_{1, n} \mid h_{2, n}\right\rangle=0, n \in \mathbb{N}$. Moreover, it follows from the above relations and the properties of Si that

$$
\lim _{n \rightarrow \infty}\left\langle h_{1, n} \mid h_{1, n}\right\rangle=\lim _{n \rightarrow \infty}\left\langle h_{2, n} \mid h_{2, n}\right\rangle=i \lim _{n \rightarrow \infty}\left\langle h_{1, n} \mid h_{2, n}\right\rangle=2 \pi \int d p \theta\left(p_{0}\right) \delta\left(p^{2}\right)|\widetilde{h}(p)|^{2},
$$

where one recalls that $\widetilde{h}(0)=0$, so the integral exists also in $d=2$ dimensions. Choosing a function $h$ such that this integral is different from 0 , one concludes that the sequence of pairs $\left\{f_{1, n} \doteq\left\langle h_{1, n} \mid h_{1, n}\right\rangle^{-1 / 2} h_{1, n}, f_{2, n} \doteq\left\langle h_{2, n} \mid h_{2, n}\right\rangle^{-1 / 2} h_{2, n}\right\}_{n \in \mathbb{N}}$ has all properties stated in the first parts of the lemma. The last assertion of the lemma is an immediate consequence of the relations

$$
\begin{aligned}
& \left\langle h_{1, n} \mid g\right\rangle=-\left(2 \pi i / n^{d-2}\right) \int d p \theta\left(p_{0}\right) \delta\left(p^{2}-m^{2}\right) \operatorname{Si}\left(p_{0} / 2 n\right) \overline{\widetilde{h}\left(p / n^{2}\right)} \widetilde{g}(p), \\
& \left\langle h_{2, n} \mid g\right\rangle=\left(2 \pi / n^{d-2}\right) \int d p \theta\left(p_{0}\right) \delta\left(p^{2}-m^{2}\right) \overline{\widetilde{h}\left(p / n^{2}\right)} \widetilde{g}(p),
\end{aligned}
$$

using in $d=2$ dimensions once more the fact that $\widetilde{h}(0)=0$.

In a last preparatory step we show that the sequences $\left\{f_{1, n}, f_{2, n}\right\}_{n \in \mathbb{N}}$ in the preceding lemma can be used to construct sequences $\left\{g_{1, n}, g_{2, n}\right\}_{n \in \mathbb{N}}$ such that the resulting pairs satisfy the condition in Lemma 3.1.

Lemma 3.3 Let $\left\{f_{1, n}, f_{2, n}\right\}_{n \in \mathbb{N}}$ be sequences of test functions as in the preceding lemma. There exists a dense set of translations $a \in \mathbb{R}^{d}$ such that for any given a in this set the corresponding sequences $\left\{\tau_{a} f_{1, n}, \tau_{a} f_{2, n}\right\}_{n \in \mathbb{N}}$, where $\left(\tau_{a} f\right)(x) \doteq f(x-a)$, satisfy

$$
\left\langle f_{1, n}+i f_{2, n} \mid \tau_{a}\left(f_{1, n}-i f_{2, n}\right)\right\rangle \neq 0, \quad n \in \mathbb{N} .
$$

Proof. Recalling that $M$ denotes the mass shell, one has $\left(\widetilde{f_{1, n} \pm i f_{2, n}}\right) \uparrow M \neq 0$, $n \in \mathbb{N}$. In fact, since $f_{1, n} \pm i f_{2, n}$ has compact support, its Fourier transform is entire analytic and can vanish on the mass shell only on a closed set of measure 0 . Thus the wave functions $\tau_{a}\left(\widetilde{f_{1, n}-i} f_{2, n}\right) \uparrow M, a \in \mathbb{R}^{d}$, form a total set in $\mathcal{H}_{1}$ for each $n \in \mathbb{N}$. Next, observe that each function $a \mapsto\left\langle f_{1, n}+i f_{2, n} \mid \tau_{a}\left(f_{1, n}-i f_{2, n}\right)\right\rangle, n \in \mathbb{N}$, 
extends to an analytic function in the forward tube $\mathbb{R}^{d}+i V_{+}$as a consequence of the relativistic spectrum condition. Hence, by the Edge-of-the-Wedge Theorem and the preceding remarks, it can vanish only on a closed, nowhere dense subset $S_{n} \subset \mathbb{R}^{d}$ for each $n \in \mathbb{N}$. Thus $\bigcup_{n \in \mathbb{N}} S_{n}$ is a meager set in the Baire space $\mathbb{R}^{d}$. Its complement is therefore dense, proving the statement.

We are now in a position to prove that the net $\mathcal{R}$ defined in this model is maximally nonlocal. As a matter of fact, the following somewhat stronger statement holds.

Proposition 3.4 Let $\mathcal{O}_{1}$ and $\mathcal{O}_{2}$ be spacelike separated regions in Minkowski space. Then $C\left(\mathcal{R}_{e}\left(\mathcal{O}_{1}\right), \mathcal{R}_{e}\left(\mathcal{O}_{2}\right)\right)=1$, where $\mathcal{R}_{e}$ denotes the subnet generated by all even polynomials in the smeared field $\phi$.

Proof. Because of the Poincaré covariance of the net, one may assume that $\mathcal{O}_{1}$ contains the point 0 in its interior. Choosing some suitable $a \in \mathbb{R}^{d}$, it then follows from the preceding Lemma that for sufficiently large $n \in \mathbb{N}$, the supports of the functions $f_{1, n}, f_{2, n}$ constructed in Lemma 3.1 are contained in $\mathcal{O}_{1}$, those of $\tau_{a} f_{1, n}, \tau_{a} f_{2, n}$ in $\mathcal{O}_{2}$, and $\left\langle f_{1, n}+i f_{2, n} \mid \tau_{a}\left(f_{1, n}-i f_{2, n}\right)\right\rangle \neq 0$ for all $n \in \mathbb{N}$. In view of the support properties of $f_{1, n}, f_{2, n}$, the projections $P_{n}=\frac{1}{2}\left(1+i \phi\left(f_{1, n}\right) \phi\left(f_{2, n}\right)\right)$ are contained in $\mathcal{R}_{e}\left(\mathcal{O}_{1}\right)$ and the projections $Q_{n}=\frac{1}{2}\left(1+i \phi\left(\tau_{a} f_{1, n}\right) \phi\left(\tau_{a} f_{2, n}\right)\right)$ in $\mathcal{R}_{e}\left(\mathcal{O}_{2}\right)$, provided $n \in \mathbb{N}$ is sufficiently large. Moreover, as a consequence of Lemma 3.1 and the fact that the above scalar products are different from 0 , one has $P_{n} \wedge Q_{n}=0$, $n \in \mathbb{N}$.

Bearing in mind that the scalar products of the one-particle states are invariant under translations, the vacuum expectation values of $P_{n}$ and $Q_{n}$ are given by $\left\langle\Omega, P_{n} \Omega\right\rangle=\left\langle\Omega, Q_{n} \Omega\right\rangle=\frac{1}{2}\left(1+i\left\langle f_{1, n} \mid f_{2, n}\right\rangle\right)$. Thus these expectation values converge to 1 in the limit of large $n$ according to Lemma 3.2 , and consequently $P_{n} \Omega \rightarrow \Omega$, $Q_{n} \Omega \rightarrow \Omega$ strongly. Moreover,

$$
\begin{aligned}
& \sup _{E, F}|\langle\Omega, E \wedge F \Omega\rangle-\langle\Omega, E \Omega\rangle\langle\Omega, F \Omega\rangle| \\
& \geq \lim _{n \rightarrow \infty}\left|\left\langle\Omega, P_{n} \wedge Q_{n} \Omega\right\rangle-\left\langle\Omega, P_{n} \Omega\right\rangle\left\langle\Omega, Q_{n} \Omega\right\rangle\right|=1,
\end{aligned}
$$

where the supremum extends over all projections $E \in \mathcal{R}_{e}\left(\mathcal{O}_{1}\right)$ and $F \in \mathcal{R}_{e}\left(\mathcal{O}_{2}\right)$. In order to see that this lower bound holds for all normal states, one makes use of the fact that the projections $P_{n}, Q_{n}$ form central sequences. To verify this, note that for each real test function $g$ one obtains by a straightforward application of the anticommutation relations the bound

$$
\left\|\left[P_{n}, \phi(g)\right]\right\| \leq\left|\left\langle f_{1, n} \mid g\right\rangle\right|\left\|\phi\left(f_{2, n}\right)\right\|+\left|\left\langle f_{2, n} \mid g\right\rangle\right|\left\|\phi\left(f_{1, n}\right)\right\| \text {. }
$$

The right hand side of this inequality tends to 0 for large $n$ according to Lemma 3.2 , Since $P_{n} \Omega \rightarrow \Omega$, strongly, and $\Omega$ is cyclic for the algebra of all polynomials in the smeared fields, it follows that $P_{n} \rightarrow 1$ in the strong operator topology on $\mathcal{H}$. By the 
same reasoning one sees that $Q_{n} \rightarrow 1$ as well. Hence, recalling that $P_{n} \wedge Q_{n}=0$, one obtains for any normal state $\omega$

$$
\sup _{E, F}|\omega(E \wedge F)-\omega(E) \omega(F)| \geq \lim _{n \rightarrow \infty}\left|\omega\left(P_{n} \wedge Q_{n}\right)-\omega\left(P_{n}\right) \omega\left(Q_{n}\right)\right|=1 .
$$

Since, from the outset, $C\left(\mathcal{R}_{e}\left(\mathcal{O}_{1}\right), \mathcal{R}_{e}\left(\mathcal{O}_{2}\right)\right) \leq 1$, the statement follows.

Another expression of the nonlocality of the present model is the absence of any nontrivial operator which commutes with all operators in the spacelike complement of its localization region. We exhibit this fact in the following Proposition.

Proposition 3.5 Let $W \in \mathcal{W}$ be any wedge region. Then $\mathcal{R}(W)^{\prime} \cap \mathcal{R}\left(W^{\prime}\right)=\mathbb{C} \cdot 1$.

Proof. It follows from Lemma 2.1 that $\mathcal{R}\left(W^{\prime}\right) \subset \widehat{\mathcal{R}}(W)^{\prime}$ and consequently $\mathcal{R}(W)^{\prime} \cap \mathcal{R}\left(W^{\prime}\right) \subset \mathcal{R}(W)^{\prime} \cap \widehat{\mathcal{R}}(W)^{\prime} \doteq \mathcal{I}(W)$. The $*$-algebra $\mathcal{I}(W)$ is invariant under the adjoint action of the unitary involutions $V, Z$, as well as of the unitaries representing the boosts which leave the wedge $W$ invariant. Let $E \in \mathcal{I}(W)$ be any even element under the adjoint action of $Z$, i.e. $E Z=E Z$, and let $f$ be any test function with support in $W$. It then follows from the results in Sect. 2 that

$$
\begin{aligned}
& 0=[E, \phi(f)]=\left[E, \phi_{+}(f)+\phi_{-}(f)\right], \\
& 0=[V E V, \phi(f)]=V\left[E,\left(\phi_{+}(f)-\phi_{-}(f)\right) Z\right] V=V\left[E,\left(\phi_{+}(f)-\phi_{-}(f)\right)\right] Z V,
\end{aligned}
$$

and consequently $\left[E, \phi_{ \pm}(f)\right]=0$ whenever supp $f \subset W$. As the operator valued functions $\mathbb{R}^{d} \ni a \mapsto\left[E, \phi_{ \pm}\left(\tau_{a} f\right)\right]$ can be continued analytically into the forward and backward tubes, respectively, and the translated test functions $\tau_{a} f$ have support in $W$ for an open set of translations $a \in \mathbb{R}^{d}$, it follows from the Edge-of-the-Wedge Theorem that $\left[E, \phi_{ \pm}(f)\right]=0$ for all test functions $f$. Since the creation and annihilation operators act irreducibly on the Fock space $\mathcal{H}, E=c \cdot 1$ for some $c \in \mathbb{C}$.

Next, let $O_{1}, O_{2} \in \mathcal{I}(W)$ be two odd elements under the adjoint action of $Z$, i.e. $O_{j} Z=-Z O_{j}, j=1,2$. Then $O_{1} O_{2}$ is even and consequently $O_{1} O_{2}=c \cdot 1$ for some $c \in \mathbb{C}$. Let $v(t), t \in \mathbb{R}$, be the one-parameter group of boosts leaving the wedge $W$ invariant. Since $Z$ commutes with all Poincaré transformations, one may replace in the preceding equation the operator $\mathrm{O}_{2}$ by $\mathrm{O}_{2}(t) \doteq U(v(t)) \mathrm{O}_{2} U(v(t))^{-1}$, giving $O_{1} O_{2}(t)=c(t) \cdot 1$, and consequently

$$
O_{1} U(v(t)) O_{2} O_{2}^{*} \Omega=O_{1} O_{2}(t) O_{2}^{*}(t) \Omega=c(t) O_{2}^{*}(t) \Omega=c(t) U(v(t)) O_{2}^{*} \Omega, \quad t \in \mathbb{R} .
$$

Now $\mathrm{w}^{-} \lim _{t \rightarrow \infty} U(v(t)) \Psi=\langle\Omega, \Psi\rangle \Omega$ for any $\Psi \in \mathcal{H}$, so the preceding equality implies $\left\langle\Omega, O_{2} O_{2}^{*} \Omega\right\rangle O_{1} \Omega=c(\infty)\left\langle\Omega, O_{2}^{*} \Omega\right\rangle \Omega$, where $c(\infty) \doteq \lim _{t \rightarrow \infty} c(t)$ must exist, since the other limits in the above equation exist. Since $O_{2}$ is odd and $\Omega$ is invariant under the action of $Z$, one has $\left\langle\Omega, O_{2}^{*} \Omega\right\rangle=0$, so either $O_{1} \Omega=0$ or $O_{2}^{*} \Omega=0$. Since $\Omega$ is separating for $\mathcal{I}(W)$ (it is cyclic for $\mathcal{R}(W)$, as can be proven by standard arguments $[1,17,28])$, one concludes that there are no nonzero odd 
elements in $\mathcal{I}(W)$. But any element of $\mathcal{I}(W)$ can be decomposed into the sum of an even and an odd one under the action of $Z$, so $\mathcal{I}(W)=\mathbb{C} \cdot 1$, proving the assertion for the net $\mathcal{R}$.

Although the present model is nonlocal in a very strong sense, we nonetheless want to uncover in the subsequent sections some interesting properties which are consistent with Einstein causality. As we shall see, the model has many features which one normally attributes to local theories.

\section{Modular structure and weak locality}

We determine in this section the modular groups associated with the vacuum vector $\Omega$ and the algebras $\mathcal{R}(W)$ corresponding to wedge regions $W \in \mathcal{W}$. It will turn out that they coincide with those found in local theories by Bisognano and Wichmann $[2,3]$; however, the modular conjugations differ from those of local theories. The results will allow us to establish a weak form of locality of wedge algebras.

We begin by noting that, within the framework given above, standard arguments $[1,17,28]$ entail that both nets $\mathcal{R}, \widehat{\mathcal{R}}$ are irreducible and that $\Omega$ is cyclic for both $\mathcal{R}(\mathcal{O})$ and $\widehat{\mathcal{R}}(\mathcal{O})$, for any nonempty open $\mathcal{O}$. Lemma 2.1 then implies that $\Omega$ is also separating for $\mathcal{R}(\mathcal{O})$ and $\widehat{\mathcal{R}}(\mathcal{O})$, whenever $\mathcal{O}^{\prime}$ is open and nonempty. Thus, in particular, $\Omega$ is cyclic and separating for all wedge algebras $\mathcal{R}(W), \widehat{\mathcal{R}}(W)$, $W \in \mathcal{W}$. The Tomita-Takesaki modular theory, cf. $[6,18]$, is therefore applicable to the pairs $(\mathcal{R}(W), \Omega),(\widehat{\mathcal{R}}(W), \Omega)$, for all wedges $W$.

It is our aim to compute the corresponding modular objects. This task is greatly facilitated by making use of known results $[2,7,17]$. Let $v_{R}(t), t \in \mathbb{R}$, be the boost subgroup in $\mathcal{P}_{+}^{\uparrow}$ which induces a positive timelike flow on the wedge $W_{R}$ and which is periodic for imaginary $t$ with period $2 \pi$. One then has

$$
v_{R}(i \pi)=\theta_{R},
$$

where $\theta_{R} \in \mathcal{P}_{+}$(the proper Poincaré group) is the reflection about the edge of $W_{R}$. Now if $f \in \mathcal{S}\left(\mathbb{R}^{d}\right)$ has support in $W_{R}$, then $|f\rangle$ is known to lie in the domain of the positive self-adjoint operator $U\left(v_{R}(i \pi)\right)$ and

$$
U\left(v_{R}(i \pi)|f\rangle=\left|\theta_{R} f\right\rangle\right.
$$

where $\left(\theta_{R} f\right)(x) \doteq f\left(\theta_{R} x\right)$. Moreover, the antilinear map $|f\rangle \mapsto\left|\theta_{R} \bar{f}\right\rangle$ is isometric. Using the functorial character of the representation $U$, these statements can be extended to the many particle states. One has

$$
U\left(v_{R}(i \pi)\right)\left|f_{1}\right\rangle \wedge \cdots \wedge\left|f_{n}\right\rangle=\left|\theta_{R} f_{1}\right\rangle \wedge \cdots \wedge\left|\theta_{R} f_{n}\right\rangle,
$$

provided all functions $f_{1}, \ldots, f_{n}$ have support in $W_{R}$; the operator $U\left(\theta_{R}\right)$ fixed by

$$
U\left(\theta_{R}\right)\left|f_{1}\right\rangle \wedge \cdots \wedge\left|f_{n}\right\rangle \doteq\left|\theta_{R} \overline{f_{1}}\right\rangle \wedge \cdots \wedge\left|\theta_{R} \overline{f_{n}}\right\rangle
$$


is an antiunitary involution extending the representation $U$ of $\mathcal{P}_{+}^{\uparrow}$ to a representation of $\mathcal{P}_{+}$. (This statement amounts to the PCT-theorem in even dimensions $d$.) Taking into account the antisymmetry properties of the many particle states, one has

$$
\left|f_{n}\right\rangle \wedge \cdots \wedge\left|f_{1}\right\rangle=(-1)^{n(n-1) / 2}\left|f_{1}\right\rangle \wedge \cdots \wedge\left|f_{n}\right\rangle=V\left|f_{1}\right\rangle \wedge \cdots \wedge\left|f_{n}\right\rangle
$$

where $V$ is the unitary operator introduced in Section 2. Combining the above relations, one arrives at

$$
V U\left(\theta_{R}\right) U\left(v_{R}(i \pi)\right)\left|f_{1}\right\rangle \wedge \cdots \wedge\left|f_{n}\right\rangle=\left|\overline{f_{n}}\right\rangle \wedge \cdots \wedge\left|\overline{f_{1}}\right\rangle
$$

provided $f_{1}, \ldots, f_{n}$ have the support properties stated above.

It is straightforward to restate these results in terms of the field operators. Making repeated use of relation (2.1), one gets

$$
\phi\left(f_{1}\right) \cdots \phi\left(f_{n}\right) \Omega=\sum_{p}(-1)^{\tau(p)}\left\langle\overline{f_{p_{1}}} \mid f_{p_{2}}\right\rangle \cdots\left\langle\overline{f_{p_{k-1}}} \mid f_{p_{k}}\right\rangle \cdot\left|f_{1}\right\rangle \cdots \stackrel{p_{1}}{\vee} \cdots \stackrel{p_{k}}{\vee} \cdots\left|f_{n}\right\rangle
$$

where the sum extends over all ordered pairs $p \subset\{1, \ldots, n\}$ and $\tau(p)$ is the number of transpositions needed to transform $1, \ldots, n$ into $p_{1}, \ldots, p_{k}, 1, \ldots \vee \ldots p^{p_{k}} \ldots, n$. Noticing that this number is equal (modulo 2) to the number of transpositions needed to transform $n, \ldots, 1$ into $p_{k}, \ldots, p_{1}, n, \ldots \vee p_{k} \ldots p_{1} \ldots, 1$ and bearing in mind that $U\left(\theta_{R}\right)$ is antilinear, one obtains with $f_{1}, \ldots, f_{n}$ as above

$$
\begin{aligned}
& V U\left(\theta_{R}\right) U\left(v_{R}(i \pi)\right) \phi\left(f_{1}\right) \cdots \phi\left(f_{n}\right) \Omega \\
& =\sum_{p}(-1)^{\tau(p)}\left\langle\overline{\overline{f_{p_{k}}}} \mid \overline{f_{p_{k-1}}}\right\rangle \cdots\left\langle\overline{\overline{f_{p_{2}}}} \mid \overline{f_{p_{1}}}\right\rangle \cdot\left|\overline{f_{n}}\right\rangle \cdots \vee p_{k} \ldots \vee p_{1} \ldots\left|\overline{f_{1}}\right\rangle \\
& =\phi\left(\overline{f_{n}}\right) \cdots \phi\left(\overline{f_{1}}\right) \Omega=\left(\phi\left(f_{1}\right) \cdots \phi\left(f_{n}\right)\right)^{*} \Omega .
\end{aligned}
$$

Since the polynomials in the fields $\phi(f)$ with $\operatorname{supp} f \subset W_{R}$ are weakly dense in $\mathcal{R}\left(W_{R}\right)$ and generate from the vacuum vector $\Omega$ a domain of essential selfadjointness for $U\left(v_{R}(i \pi)\right)$, it follows that $S_{R}=V U\left(\theta_{R}\right) U\left(v_{R}(i \pi)\right)$ is the Tomita conjugation for the pair $\left(\mathcal{R}\left(W_{R}\right), \Omega\right)$. As the expression given for $S_{R}$ is already its polar decomposition, it is apparent that $\Delta_{R}=U\left(v_{R}(2 i \pi)\right)$ is the modular operator and $J_{R}=V U\left(\theta_{R}\right)$ the modular conjugation associated with $\left(\mathcal{R}\left(W_{R}\right), \Omega\right)$. Making use of relations (2.1) and (4.1), one checks that $J_{R} \phi(f) J_{R}=V \phi\left(\theta_{R} \bar{f}\right) V=$ $\widehat{\phi}\left(\theta_{R} \bar{f}\right)$. Thus $\mathcal{R}\left(W_{R}\right)^{\prime}=J_{R} \mathcal{R}\left(W_{R}\right) J_{R}=\widehat{\mathcal{R}}\left(W_{R}^{\prime}\right)$, where the first equality follows from Tomita-Takesaki theory and the second one from the geometrical fact that $\theta_{R} W_{R}=W_{L}=W_{R}{ }^{\prime}$. Since the Poincaré transformations commute with $V$, the modular objects corresponding to $\left(\widehat{\mathcal{R}}\left(W_{R}\right), \Omega\right)$ coincide with those of $\left(\mathcal{R}\left(W_{R}\right), \Omega\right)$, and one has $\widehat{\mathcal{R}}\left(W_{R}\right)^{\prime}=J_{R} \widehat{\mathcal{R}}\left(W_{R}\right) J_{R}=\mathcal{R}\left(W_{R}{ }^{\prime}\right)$. We summarize these results in the following proposition. 
Proposition 4.1 The modular operator and conjugation corresponding to the pair $\left(\mathcal{R}\left(W_{R}\right), \Omega\right)$ are given by $\Delta_{R}=U\left(v_{R}(2 i \pi)\right)$ and $J_{R}=V U\left(\theta_{R}\right)$, respectively. Moreover,

$$
\mathcal{R}\left(W_{R}\right)^{\prime}=\widehat{\mathcal{R}}\left(W_{R}{ }^{\prime}\right) .
$$

These statements hold likewise for $\left(\widehat{\mathcal{R}}\left(W_{R}\right), \Omega\right)$, if one interchanges $\mathcal{R}$ and $\widehat{\mathcal{R}}$ in the preceding equality.

Since the proper Poincaré group $\mathcal{P}_{+}$acts covariantly and transitively on the wedge algebras, this result extends to the pairs $(\mathcal{R}(W), \Omega)$ for arbitrary wedge regions $W$ in an obvious manner. In particular, the corresponding modular groups are induced by the boosts leaving the respective wedge $W$ invariant. This property of a net has come to be called Modular Covariance, cf. the review article [4]. It readily follows that this model satisfies the superstability conditions studied in [11]. However, since the net is not local, the modular conjugations do not act in a geometric manner, i.e. the Condition of Geometric Modular Action [9] is not satisfied in this model. Nonetheless, the modular structure exhibited above allows one to establish a rudiment of locality which prima facie is stronger than the property of "weak locality" established for the present model in [17].

Proposition 4.2 Let $W$ be any wedge region. Then

$$
\langle\Omega, A B \Omega\rangle=\langle\Omega, B A \Omega\rangle \quad \text { for } A \in \mathcal{R}(W), B \in \mathcal{R}\left(W^{\prime}\right) .
$$

Proof. It suffices to prove this statement for the wedge $W_{R}$. Since $\mathcal{R}\left(W_{R}{ }^{\prime}\right)=$ $\widehat{\mathcal{R}}\left(W_{R}\right)^{\prime}$, the modular objects corresponding to $\left(\mathcal{R}\left(W_{R}{ }^{\prime}\right), \Omega\right)$ are given by $\Delta_{R}^{-1}, J_{R}$. So one has

$$
\begin{aligned}
& \langle\Omega, A B \Omega\rangle=\left\langle A^{*} \Omega, B \Omega\right\rangle=\left\langle J_{R} \Delta_{R}^{1 / 2} A \Omega, J_{R} \Delta_{R}^{-1 / 2} B^{*} \Omega\right\rangle \\
& =\left\langle\Delta_{R}^{-1 / 2} B^{*} \Omega, \Delta_{R}^{1 / 2} A \Omega\right\rangle=\langle\Omega, B A \Omega\rangle,
\end{aligned}
$$

as claimed.

\section{$5 \quad$ Independence properties}

The preceding information on the modular operators corresponding to wedge algebras and the vacuum state allows one to establish strong independence properties of pairs of such algebras associated with spacelike separated wedge regions. Our first result says that any pair of such algebras has no nontrivial operator in common. This fact is, in a sense, complementary to the statement of Proposition 3.5. The proof is based on standard arguments, cf. [11], which we recall here for the convenience of the reader.

Proposition 5.1 For any wedge $W \in \mathcal{W}$, one has $\mathcal{R}(W) \cap \mathcal{R}\left(W^{\prime}\right)=\mathbb{C} \cdot 1$. 
Proof. Because of covariance, it suffices to prove the statement for the wedge $W_{R}$. Now according to Proposition 4.1, $J_{R}, \Delta_{R}$ are the modular objects corresponding to $\left(\mathcal{R}\left(W_{R}\right), \Omega\right)$, and $J_{R}, \Delta_{R}^{-1}$ are those corresponding to $\left(\mathcal{R}\left(W_{R}^{\prime}\right)=\widehat{\mathcal{R}}\left(W_{R}\right)^{\prime}, \Omega\right)$. Thus for any $A \in \mathcal{R}\left(W_{R}\right) \cap \mathcal{R}\left(W_{R}^{\prime}\right)$ one has $J_{R} \Delta_{R}^{1 / 2} A \Omega=A^{*} \Omega=J_{R} \Delta_{R}^{-1 / 2} A \Omega$, and consequently $\Delta_{R} A \Omega=A \Omega$. But this implies $A \Omega=\Delta_{R}^{i t} A \Omega=U\left(v_{R}(2 \pi t)\right) A \Omega$, $t \in \mathbb{R}$. Proceding in this equality to the limit $t \rightarrow \infty$, one obtains $A \Omega=\langle\Omega, A \Omega\rangle \Omega$. Since $\Omega$ is separating for $\mathcal{R}\left(W_{R}\right) \cap \mathcal{R}\left(W_{R}^{\prime}\right)$, the assertion follows.

For strictly spacelike separated wedges $W_{1}, W_{2} \in \mathcal{W}$, i.e. $\overline{W_{1}} \subset W_{2}^{\prime}$, one can establish a substantially stronger variant of this result, which expresses the algebraic independence of the corresponding wedge algebras. In local quantum field theory, an analogous result was proven by Schlieder [25] and Roos [24], using quite different arguments.

Proposition 5.2 Let $W_{1}, W_{2} \in \mathcal{W}$ be strictly spacelike separated. For any $n \in \mathbb{N}$, $A_{1, k} \in \mathcal{R}\left(W_{1}\right)$ and $A_{2, k} \in \mathcal{R}\left(W_{2}\right), k=1, \ldots, n$, such that $\sum_{k=1}^{n} A_{1, k} A_{2, k}=0$, one must have

$$
\sum_{k=1}^{n} \psi\left(A_{1, k}\right) A_{2, k}=0=\sum_{k=1}^{n} A_{1, k} \psi\left(A_{2, k}\right),
$$

for all normal linear functionals $\psi$ on $\mathcal{B}(\mathcal{H})$. In particular, if $A_{1} A_{2}=0$, then either $A_{1}=0$ or $A_{2}=0$.

Proof. Since the proof follows in the steps of the arguments given in $[11$, Sect. III], only the necessary changes to be made will be indicated.

Due to Poincaré covariance, there is no loss of generality to assume that $\overline{W_{1}} \subset$ $W_{R}$ and $\overline{W_{2}} \subset W_{R}^{\prime}$. Let $\mathcal{P}_{R} \subset \mathcal{P}_{+}^{\uparrow}$ be the subgroup generated by the boosts $v_{R}(\mathbb{R})$, which leave the wedge $W_{R}$ invariant, and the two-dimensional subspace of spacetime translations which are orthogonal to the edge of $W_{R}$; thus $\mathcal{P}_{R}$ is isomorphic to the identity component of the Poincaré group in two dimensions. The following facts enter into the proof: (a) There is an open neighborhood $\mathcal{N}_{R} \subset$ $\mathcal{P}_{R}$ of the identity such that $W_{1} \subset \lambda_{0} \lambda_{1} W_{R}$ and $W_{2} \subset \lambda_{0} \lambda_{1} W_{R}^{\prime}$ for all $\lambda_{0}, \lambda_{1} \in \mathcal{N}_{R}$. (b) The group generated by $\left\{\lambda v_{R}(t) \lambda^{-1}: t \in \mathbb{R}, \lambda \in \mathcal{N}_{R}\right\}$ coincides with $\mathcal{P}_{R}$. (c) Making use of Proposition (4.1) and the irreducibility of the net $\widehat{\mathcal{R}}$, one has

$$
\bigvee_{\lambda \in \mathcal{P}_{R}} U(\lambda) \mathcal{R}\left(W_{R}\right)^{\prime} U(\lambda)^{-1}=\bigvee_{\lambda \in \mathcal{P}_{R}} U(\lambda) \widehat{\mathcal{R}}\left(W_{R}^{\prime}\right) U(\lambda)^{-1}=\mathcal{B}(\mathcal{H})
$$

Taking into account that $t \mapsto U\left(v_{R}(2 \pi t)\right)$ and $t \mapsto U\left(v_{R}(-2 \pi t)\right)$ are the modular groups corresponding to $\left(\mathcal{R}\left(W_{R}\right), \Omega\right)$ and $\left(\mathcal{R}\left(W_{R}\right)^{\prime}, \Omega\right)$, respectively, the necessary ingredients for the arguments given in [11] are therefore in place here, as well. More specifically, the statements and proofs of [11, Lemma 3.1, Lemma 3.2] carry over to the present situation, if one replaces the group $S O(2, n-1)$ by $\mathcal{P}_{R}$. The assertion then follows as in [11, Proposition 3.3]. 


\section{Coherent families of local observables}

As nonlocal as we have seen the net $\{\mathcal{R}(W)\}_{W \in \mathcal{W}}$ to be, nonetheless it accomodates quantities which one may assign to point-, string- or brane-shaped regions and which commute when spatially separated. In this section we shall exhibit explicit examples of such operators.

Let $W_{1}, W_{2} \in \mathcal{W}$ be wedges such that $\bar{W}_{2} \subset W_{1}$. Because of Poincaré covariance, we may assume that $W_{1}=W_{R}$ and $W_{2}=W_{R}+(a, 0, \ldots 0)$ for some $a>0$. So both edges of these wedges lie in the time-0-plane. We want to show that there exist nontrivial field operators $\phi(h) \in \mathcal{R}\left(W_{1}\right)$ which anticommute with all field operators $\phi(f) \in \mathcal{R}\left(W_{2}\right)$. To this end, we need the following preparatory lemma.

Lemma 6.1 Let $a>0$. There exist test functions $\widetilde{k} \in \mathcal{S}\left(\mathbb{R}^{d-1}\right)$ such that

$$
\boldsymbol{x} \mapsto k(\boldsymbol{x})=(2 \pi)^{(1-d) / 2} \int d \boldsymbol{p} \widetilde{k}(\boldsymbol{p}) e^{i \boldsymbol{p} \boldsymbol{x}}
$$

vanishes in the region $\left\{\boldsymbol{x} \in \mathbb{R}^{d-1}: x_{1}<0\right\}$ and

$$
\boldsymbol{x} \mapsto(2 \pi)^{(1-d) / 2} \int \frac{d \boldsymbol{p}}{\sqrt{\boldsymbol{p}^{2}+m^{2}}} \widetilde{k}(\boldsymbol{p}) e^{i \boldsymbol{p} \boldsymbol{x}}
$$

vanishes in the region $\left\{\boldsymbol{x} \in \mathbb{R}^{d-1}: x_{1}>a\right\}$, respectively.

Proof. Begin by noting that for fixed $\kappa>0$ the distributions

$$
y \mapsto \int d q \sqrt{q-i \kappa} e^{i q y} \text { and } y \mapsto \int d q \frac{1}{\sqrt{q+i \kappa}} e^{i q y}
$$

vanish on $\mathbb{R}_{-}$and $\mathbb{R}_{+}$, respectively, because of the analyticity and temperedness properties of the integrands. Consider now, for given $l \in \mathcal{S}\left(\mathbb{R}^{d-1}\right)$ with compact support in $\left\{\boldsymbol{x} \in \mathbb{R}^{d-1}: 0<x_{1}<a\right\}$, the functions

$$
\widetilde{k}(\boldsymbol{p}) \doteq \sqrt{p_{1}-i \sqrt{\boldsymbol{p}_{\perp}^{2}+m^{2}}} \widetilde{l}(\boldsymbol{p}), \quad \frac{1}{\sqrt{\boldsymbol{p}^{2}+m^{2}}} \widetilde{k}(\boldsymbol{p})=\frac{\widetilde{l}(\boldsymbol{p})}{\sqrt{p_{1}+i \sqrt{\boldsymbol{p}_{\perp}^{2}+m^{2}}}}
$$

where $\boldsymbol{p}_{\perp}$ is the component of $\boldsymbol{p}$ which is orthogonal to the 1-direction. Since the Fourier transform of a product of functions coincides with the convolution of the Fourier transforms of the individual factors, the assertion follows.

Since $\|\phi(g)\|^{2} \leq\langle g \mid g\rangle+\langle\bar{g} \mid \bar{g}\rangle$, the field $\phi$ can be extended by continuity to (generalized) functions of the form $x \mapsto h(x)=\delta\left(x_{0}\right) k(\boldsymbol{x})$, where $k$ is any test function as in the preceding lemma. Because of the support properties of $k$ and the fact that $\mathcal{R}\left(W_{1}\right)$ is norm-closed, it follows that $\phi(h) \in \mathcal{R}\left(W_{1}\right)$. Now the function

$$
x \mapsto\{\phi(h), \phi(x)\}=(2 \pi)^{(1-d) / 2} \int \frac{d \boldsymbol{p}}{\sqrt{\boldsymbol{p}^{2}+m^{2}}} \tilde{k}(\boldsymbol{p}) \cos \left(x_{0} \sqrt{\boldsymbol{p}^{2}+m^{2}}\right) e^{i \boldsymbol{p} \boldsymbol{x}} \cdot 1
$$


is a solution of the Klein-Gordon equation whose Cauchy data at time $x_{0}=0$ vanish in the region $\left\{\boldsymbol{x} \in \mathbb{R}^{d-1}: x_{1}>a\right\}$ according to the preceding lemma. Because of the hyperbolic nature of the Klein-Gordon equation, this function thus vanishes also in the causal completion of that region, i.e. in the wedge $W_{2}$. Thus we conclude that $\{\phi(h), \phi(f)\}=0$ for all test functions $f$ with supp $f \subset W_{2}$.

We finally note that the Fourier transforms of the functions $h$ defined above can vanish on the mass shell $M$ only on subsets of measure 0 , since the functions $l$ entering in the preceding lemma in the construction of $k$ have compact supports. As a consequence, $\Omega$ is cyclic for the algebra generated by the field operators $\left\{\phi\left(\tau_{a} h\right): a \in N\right\}$, where $N \subset \mathbb{R}^{d}$ is any open neighborhood of the origin. So we have established the following result.

Lemma 6.2 Let $W_{1}, W_{2} \in \mathcal{W}$ be wedges such that $\overline{W_{2}} \subset W_{1}$. Then there exist nonzero elements $\phi(h) \in \mathcal{R}\left(W_{1}\right)$ such that

$$
\{\phi(h), \phi(f)\}=0 \quad \text { whenever } \operatorname{supp} f \subset W_{2} .
$$

Indeed, there are so many such elements that $\Omega$ is a cyclic vector for the algebra they generate.

We make use of this result in order to define nontrivial algebras of local operators, which can be assigned to the intersection of certain specific wedge regions. We begin with the following definition.

Definition Let $W_{0} \in \mathcal{W}$ be a fixed wedge. (a) A wedge $W \in \mathcal{W}$ is said to be coherent with $W_{0}$ if there exists some translation $a \in \mathbb{R}^{d}$ such that $W+a \subset W_{0}$; the set of all wedges which are coherent with $W_{0}$ is denoted by $\mathcal{W}_{0}$. (b) The subgroup of $\mathcal{P}_{+}^{\uparrow}$ generated by the translations $\mathbb{R}^{d}$ and the stability group of $W_{0}$ in $\mathcal{P}_{+}^{\uparrow}$ is denoted by $\mathcal{P}_{0}$; it is the largest subgroup of $\mathcal{P}_{+}^{\uparrow}$ whose action leaves the set $\mathcal{W}_{0}$ of coherent wedges invariant.

Fixing a wedge $W_{0}$ and corresponding coherent family $\mathcal{W}_{0}$ as above, we assign to each pair of wedges $W_{1}, W_{2} \in \mathcal{W}_{0}$ such that $\overline{W_{2}} \subset W_{1}$, i.e. $W_{1} \cap W_{2}^{\prime}$ has nonempty interior, the algebra

$$
\mathcal{A}_{0}\left(W_{1} \cap W_{2}^{\prime}\right) \doteq \mathcal{R}\left(W_{1}\right) \cap \mathcal{R}\left(W_{2}\right)^{\prime} .
$$

As an immediate consequence of the preceding lemma, all even polynomials of the operators $\phi(h)$ described there are elements of this algebra, so it is clearly nontrivial. If $W_{3}, W_{4} \in \mathcal{W}_{0}$ are such that $W_{1} \cap W_{2}^{\prime} \subset W_{3} \cap W_{4}^{\prime}$, it follows after a moment's reflection that $W_{1} \subset W_{3}$ and $W_{4} \subset W_{2}$. Hence

$$
\mathcal{A}_{0}\left(W_{1} \cap W_{2}^{\prime}\right)=\mathcal{R}\left(W_{1}\right) \cap \mathcal{R}\left(W_{2}\right)^{\prime} \subset \mathcal{R}\left(W_{3}\right) \cap \mathcal{R}\left(W_{4}\right)^{\prime}=\mathcal{A}_{0}\left(W_{3} \cap W_{4}^{\prime}\right)
$$

since the net $\mathcal{R}$ is inclusion preserving. If, on the other hand, the regions $W_{1} \cap W_{2}^{\prime}$ and $W_{3} \cap W_{4}^{\prime}$ are spacelike separated, then either $W_{1} \subset W_{4}$ or $W_{3} \subset W_{2}$. Hence, in either case,

$$
\mathcal{A}_{0}\left(W_{1} \cap W_{2}^{\prime}\right)=\mathcal{R}\left(W_{1}\right) \cap \mathcal{R}\left(W_{2}\right)^{\prime} \subset \mathcal{R}\left(W_{4}\right) \vee \mathcal{R}\left(W_{3}\right)^{\prime}=\mathcal{A}_{0}\left(W_{3} \cap W_{4}^{\prime}\right)^{\prime} .
$$


So the map $W_{1} \cap W_{2}^{\prime} \mapsto \mathcal{A}_{0}\left(W_{1} \cap W_{2}^{\prime}\right)$ defines a local net based on intersections of coherent wedges. Since these intersections are infinitely extended in $(d-2)$ spatial directions and arbitrarily thin in the remaining 2 directions, the underlying operators may be thought of as being point-, string- or brane-localized, respectively, if $d=2,3$ or $\geq 4$. In view of the covariant action of the Poincaré transformations on the net $\mathcal{R}$, one also has

$U\left(\lambda_{0}\right) \mathcal{A}_{0}\left(W_{1} \cap W_{2}^{\prime}\right) U\left(\lambda_{0}\right)^{-1}=\mathcal{R}\left(\lambda_{0} W_{1}\right) \cap \mathcal{R}\left(\lambda_{0} W_{2}\right)^{\prime}=\mathcal{A}_{0}\left(\lambda_{0}\left(W_{1} \cap W_{2}^{\prime}\right)\right), \quad \lambda_{0} \in \mathcal{P}_{0}$,

since $\lambda_{0} W_{1}, \lambda_{0} W_{2} \in \mathcal{W}_{0}$. We summarize these results.

Proposition 6.3 Let $\mathcal{W}_{0}$ be a coherent set of wedges. The corresponding net $\mathcal{A}_{0}$, defined in (6.2), is local, $\mathcal{P}_{0}$-covariant and nontrivial.

Thus, in spite of the nonlocality of the net $\mathcal{R}$, its net structure can be used to identify coherent families of subalgebras, which may be regarded as theories of spatially extended local operators. Knowing that these subalgebras are nontrivial, it is natural to ask how big they actually are. It is an interesting fact that the answer depends on the number $d$ of spacetime dimensions. In the formulation of the subsequent results there appear the subspace $\mathcal{H}_{e} \subset \mathcal{H}$ of states with an even particle number and the nets $\mathcal{R}_{e}, \widehat{\mathcal{R}}_{e}$ generated by even polynomials in the fields $\phi$ and $\widehat{\phi}$, respectively.

Proposition 6.4 Let $d \geq 3$, let $\mathcal{W}_{0}$ be the set of all wedges in $\mathbb{R}^{d}$ which are coherent with a given wedge $W_{0}$, and let $\overline{W_{2}} \subset W_{1} \in \mathcal{W}_{0}$. Then

(a) $\overline{\mathcal{A}_{0}\left(W_{1} \cap W_{2}^{\prime}\right) \Omega}=\mathcal{H}_{e}$,

(b) $\bigvee_{W_{1} \cap W_{2}^{\prime} \subset W_{0}} \mathcal{A}_{0}\left(W_{1} \cap W_{2}^{\prime}\right)=\mathcal{R}_{e}\left(W_{0}\right)$,

(c) $\bigvee_{W_{1} \cap W_{2}^{\prime} \subset W_{0}^{\prime}} \mathcal{A}_{0}\left(W_{1} \cap W_{2}^{\prime}\right)=\widehat{\mathcal{R}}_{e}\left(W_{0}^{\prime}\right)$.

Proof. (a) Each algebra $\mathcal{A}_{0}\left(W_{1} \cap W_{2}^{\prime}\right)$ contains all even polynomials of the field $\phi$ smeared with functions $h$ as in the statement of Lemma 6.2. It follows that $\mathcal{H}_{e} \subset$ $\overline{\mathcal{A}_{0}\left(W_{1} \cap W_{2}^{\prime}\right) \Omega}$. For the proof of the converse inclusion, note that $\mathcal{A}_{0}\left(W_{1} \cap W_{2}^{\prime}\right)$ is stable under the adjoint action of $Z=(-1)^{N}$. Hence one must show that there is no nonzero element $X \in \mathcal{A}_{0}\left(W_{1} \cap W_{2}^{\prime}\right)$ satisfying $X Z=-Z X$, i.e. being odd. To this end, one makes use of Lemma 6.2 once again and picks functions $h$ such that $\{\phi(h), \phi(f)\}=0$ whenever supp $f \subset W_{1}$. As $\mathcal{R}\left(W_{1}\right)$ is generated by the operators $\phi(f)$, it follows that $\phi(h) Z \in \mathcal{R}\left(W_{1}\right)^{\prime}$, and since $X \in \mathcal{R}\left(W_{1}\right)$ is odd, $X Z$ must commute with all operators $\phi(h)$. On the other hand, $[X, \phi(g)]=0$ whenever supp $g \subset W_{2}$, since $X \in \mathcal{R}\left(W_{2}\right)^{\prime}$.

Now let $a$ be any translation along the edge of $W_{0}$ (such translations exist if $d \geq 3$ ) and let $B(a) \doteq U(a) B U(a)^{-1}, B \in \mathcal{B}(\mathcal{H})$. In view of the invariance of 
the wedges in $\mathcal{W}_{0}$ under the action of $a$, one obtains for functions $h_{1}, \ldots h_{n}$ and $g_{1}, \ldots g_{m}$ as above the equality

$$
\begin{aligned}
& \left\langle\phi\left(g_{1}\right) \cdots \phi\left(g_{m}\right) \Omega,\left(X^{*} X Z\right)(a) \phi\left(h_{1}\right) \cdots \phi\left(h_{n}\right) \Omega\right\rangle \\
& \quad=\left\langle X(a) \Omega,\left(\phi\left(g_{m}\right)^{*} \cdots \phi\left(g_{1}\right)^{*} \phi\left(h_{1}\right) \cdots \phi\left(h_{n}\right)\right)(X Z)(a) \Omega\right\rangle \\
& =\left\langle X \Omega,\left(\phi\left(g_{m}\right)^{*} \cdots \phi\left(g_{1}\right)^{*} \phi\left(h_{1}\right) \cdots \phi\left(h_{n}\right)\right)(-a) X Z \Omega\right\rangle .
\end{aligned}
$$

Since the field $\phi$ is irreducible and the anticommutators $\{\phi(u), \phi(v)(-a)\}$ vanish in norm for arbitrary test functions $u, v$ as $a$ tends to infinity, one obtains in this limit

$$
\left(\phi\left(g_{m}\right)^{*} \cdots \phi\left(g_{1}\right)^{*} \phi\left(h_{1}\right) \cdots \phi\left(h_{n}\right)\right)(-a) \rightarrow\left\langle\Omega, \phi\left(g_{m}\right)^{*} \cdots \phi\left(g_{1}\right)^{*} \phi\left(h_{1}\right) \cdots \phi\left(h_{n}\right) \Omega\right\rangle \cdot 1
$$

in the weak operator topology. Combining these results and taking into account that $Z \Omega=\Omega$, it follows that

$$
\lim _{a \rightarrow \infty}\left(X^{*} X Z\right)(a)=\left\langle\Omega, X^{*} X \Omega\right\rangle \cdot 1
$$

and, in a similar manner,

$$
\lim _{a \rightarrow \infty}\left(X^{*} X\right)(a)=\left\langle\Omega, X^{*} X \Omega\right\rangle \cdot 1 .
$$

As $Z$ is invariant under the adjoint action of the translations, the latter two results are only compatible if $X \Omega=0$. But $\Omega$ is separating for $\mathcal{A}_{0}\left(W_{1} \cap W_{2}^{\prime}\right)$, hence $X=0$, proving that $\overline{\mathcal{A}_{0}\left(W_{1} \cap W_{2}^{\prime}\right) \Omega} \subset \mathcal{H}_{e}$.

(b) According to Proposition 4.1, the modular group corresponding to the pair $\left(\mathcal{R}\left(W_{0}\right), \Omega\right)$ consists of the unitary boost transformations leaving the wedge $W_{0}$ invariant. As the even subnet $\mathcal{R}_{e}$ is left invariant under their adjoint action, it follows that the modular group of $\left(\mathcal{R}_{e}\left(W_{0}\right), \Omega\right)$ coincides with the restriction of the boosts to the subspace $\mathcal{H}_{e}$. Since these boosts act covariantly on the net $\mathcal{A}_{0}$, the algebra $\bigvee_{W_{1} \cap W_{2}^{\prime} \subset W_{0}} \mathcal{A}_{0}\left(W_{1} \cap W_{2}^{\prime}\right) \subset \mathcal{R}_{e}\left(W_{0}\right)$ is stable under their action. But $\Omega$ is cyclic in $\mathcal{H}_{e}$ for the algebras $\mathcal{A}_{0}\left(W_{1} \cap W_{2}^{\prime}\right)$, so one must have equality in this inclusion by a standard result in modular theory. Statement (c) follows from Proposition 4.1 in a similar manner.

In a manner similar to the construction of the net $\mathcal{A}_{0}$, one can proceed from $\mathcal{R}$ to a $\mathcal{P}_{0}$-covariant field net $\mathcal{F}_{0}$ satisfying twisted locality (in analogy to theories of local Fermi fields). Introducing the twisted algebras $\mathcal{R}^{t}(\mathcal{O}) \doteq\{\phi(f) Z: \operatorname{supp} f \subset \mathcal{O}\}^{\prime \prime}$, one defines for any $\overline{W_{2}} \subset W_{1} \in \mathcal{W}_{0}$

$$
\begin{aligned}
& \mathcal{F}_{0}\left(W_{1} \cap W_{2}^{\prime}\right) \doteq \mathcal{R}\left(W_{1}\right) \cap \mathcal{R}^{t}\left(W_{2}\right)^{\prime}, \\
& \mathcal{F}_{0}^{t}\left(W_{1} \cap W_{2}^{\prime}\right) \doteq \mathcal{R}^{t}\left(W_{1}\right) \cap \mathcal{R}\left(W_{2}\right)^{\prime} .
\end{aligned}
$$

If the regions $W_{1} \cap W_{2}^{\prime}$ and $W_{3} \cap W_{4}^{\prime}$ are spacelike separated, then

$$
\mathcal{F}_{0}\left(W_{1} \cap W_{2}^{\prime}\right)=\mathcal{R}\left(W_{1}\right) \cap \mathcal{R}^{t}\left(W_{2}\right)^{\prime} \subset \mathcal{R}\left(W_{4}\right) \vee \mathcal{R}^{t}\left(W_{3}\right)^{\prime}=\mathcal{F}_{0}^{t}\left(W_{3} \cap W_{4}^{\prime}\right)^{\prime},
$$


proving twisted locality. Since $Z$ commutes with the unitaries representing the Poincaré transformations, it is also clear that the net $\mathcal{F}_{0}$ is $\mathcal{P}_{0}$-covariant. Moreover, it is an immediate consequence of Lemma 6.2 that $\overline{\mathcal{F}_{0}\left(W_{1} \cap W_{2}^{\prime}\right) \Omega}=\mathcal{H}$, so $\Omega$ is cyclic for the field net. Finally, by the preceding proposition one has for spacetime dimensions $d \geq 3$

$\mathcal{R}\left(W_{1}\right) \cap \mathcal{R}\left(W_{2}\right)^{\prime}=\mathcal{R}_{e}\left(W_{1}\right) \cap \mathcal{R}\left(W_{2}\right)^{\prime}=\mathcal{R}_{e}\left(W_{1}\right) \cap \mathcal{R}^{t}\left(W_{2}\right)^{\prime} \subset \mathcal{R}\left(W_{1}\right) \cap \mathcal{R}^{t}\left(W_{2}\right)^{\prime}$,

where the second equality follows from the fact that all elements of the net $\mathcal{R}_{e}$ commute with the unitary operator $Z$. Hence $\mathcal{A}_{0}\left(W_{1} \cap W_{2}^{\prime}\right) \subset \mathcal{F}_{0}\left(W_{1} \cap W_{2}^{\prime}\right)$ in this case. The situation is different, however, in $d=2$ spacetime dimensions.

Proposition 6.5 Let $d=2$, let $\mathcal{W}_{0}$ be the set of all wedges in $\mathbb{R}^{2}$ which are coherent with a given wedge $W_{0}$ and let $\overline{W_{2}} \subset W_{1} \in \mathcal{W}_{0}$. Then

(a) $\overline{\mathcal{A}_{0}\left(W_{1} \cap W_{2}^{\prime}\right) \Omega}=\mathcal{H}$

(b) $\bigvee_{W_{1} \cap W_{2}^{\prime} \subset W_{0}} \mathcal{A}_{0}\left(W_{1} \cap W_{2}^{\prime}\right)=\mathcal{R}\left(W_{0}\right)$,

(c) $\bigvee_{W_{1} \cap W_{2}^{\prime} \subset W_{0}^{\prime}} \mathcal{A}_{0}\left(W_{1} \cap W_{2}^{\prime}\right)=\widehat{\mathcal{R}}\left(W_{0}^{\prime}\right)$.

Thus one recovers the original states and wedge algebras from the net $\mathcal{A}_{0}$.

Proof. The crucial step in the argument is the demonstration that the vacuum vector $\Omega$ is cyclic for the algebras $\mathcal{A}_{0}\left(W_{1} \cap W_{2}^{\prime}\right)$. Instead of proving the cyclicity of $\Omega$ by abstract arguments as in [21], we explicitly exhibit sufficiently many operators in $\mathcal{A}_{0}\left(W_{1} \cap W_{2}^{\prime}\right)$. The essential ingredient is the observation [20] that the algebras $\mathcal{R}\left(W_{1}\right), \mathcal{R}\left(W_{2}\right)$ form a "split inclusion" in $d=2$ dimensions, i.e. there is a von Neumann algebra $\mathcal{M} \subset \mathcal{B}(\mathcal{H})$ of type $\mathrm{I}_{\infty}$ such that $\mathcal{R}\left(W_{2}\right) \subset \mathcal{M} \subset \mathcal{R}\left(W_{1}\right)$. It then follows from results of Doplicher and Longo [14], cf. also [8], that there exists a self-adjoint idempotent operator $Z_{1} \in \mathcal{R}\left(W_{1}\right)$ which implements the adjoint action of $Z$ on $\mathcal{R}\left(W_{2}\right)$ and satisfies $Z_{1} Z=Z Z_{1}$, i.e. it is even. Thus if $h$ is any function as in Lemma 6.2, one has $Z_{1} \phi(h) \in \mathcal{R}\left(W_{1}\right)$. On the other hand, if $\operatorname{supp} f \subset W_{2}$ then $Z_{1} \phi(h) \phi(f)=-Z_{1} \phi(f) \phi(h)=\phi(f) Z_{1} \phi(h)$ and consequently $Z_{1} \phi(h) \in \mathcal{R}\left(W_{2}\right)^{\prime}$.

Now let $\Phi \in \mathcal{H}$ be orthogonal to $\mathcal{A}_{0}\left(W_{1} \cap W_{2}^{\prime}\right) \Omega$. If $h_{1}, \ldots h_{2 n+1}$ are functions as in Lemma 6.2, then $Z_{1} \phi\left(h_{1}\right) \cdots \phi\left(h_{2 n+1}\right) \in \mathcal{A}_{0}\left(W_{1} \cap W_{2}^{\prime}\right)$ by the preceding argument and consequently $Z_{1} \Phi$ must be an element of the even subspace $\mathcal{H}_{e}$. Similarly, if $h_{1}, \ldots h_{2 n}$ are functions as in Lemma 6.2, one has $\phi\left(h_{1}\right) \cdots \phi\left(h_{2 n}\right) \in \mathcal{A}\left(W_{1} \cap W_{2}^{\prime}\right)$ and consequently $\Phi$ must lie in the subspace $\mathcal{H}_{o}$ generated by states with an odd particle number. As $Z_{1}$ is even, this is only possible if $\Phi=0$. This establishes the first part of the statement. The remaining statements then follow as in the preceding proposition.

In contrast to the situation in higher dimensions, the local and covariant net $\mathcal{A}_{0}$ has the vacuum $\Omega$ as a cyclic vector if $d=2$. In particular, there exist local operators in $\mathcal{A}_{0}$ interpolating between $\Omega$ and the single particle states. One can therefore apply Haag-Ruelle collision theory and finds [21] that the net describes 
a Boson with nontrivial scattering matrix $S=(-1)^{N(N-1) / 2}$. On the other hand, the field net $\mathcal{F}_{0}$ defined in relation (6.4) coincides with the net generated by a local free Fermi field and therefore describes a Fermion with trivial scattering matrix. Thus, in $d=2$ dimensions, the nonlocal net $\mathcal{R}$ comprises different local structures.

\section{Final Comments}

In a number of recent papers $[10,11,19-22,26,27]$, it has proven advantageous to consider nonlocal but weakly local fields for the construction of local observable algebras by purely algebraic means. Up to now, these investigations focussed on models in $d=2$ dimensions. We have therefore studied in the present paper a prototype of such models in an arbitrary number of spacetime dimensions. As we have seen, this model has many features in common with local theories. In fact, there exist nets $\mathcal{A}_{0}, \mathcal{F}_{0}$ of operators embedded in the original nonlocal net $\mathcal{R}$ which are localized in point-, string- or brane-shaped subregions of spacetime, respectively, and (anti)commute at spatial distances.

Within the general setting of algebraic quantum field theory, such partial locality properties of operators interpolating between the vacuum and single particle states are sufficient in order to establish the existence of collision states and of a corresponding scattering matrix. Moreover, this scattering matrix has the macroscopic causality (clustering) properties familiar from local field theory. We refrain from proving here these statements and refer the interested reader to [5], where the essential ingredients for a collision theory involving infinitely extended local operators can be found.

We have also seen that the net $\mathcal{R}$ is maximally nonlocal in terms of the quantitative measure $\mathcal{C}$ introduced in [12]. Nonetheless, it contains the above well-behaved local structures and manifests a number of physically desirable properties. At this stage of our investigation into locality/nonlocality in quantum field theory, this seems to be a striking fact.

In view of these findings and the growing interest in nonlocal quantum field theoretical models, it seems worthwhile to study more systematically the structure of such theories. As we have seen in Section 6, an essential step in the analysis is the determination of the relative commutants of given inclusions of algebras. In general, there is no reason for such relative commutants to be nontrivial, much less to be large. In the model at hand we have been able to determine the size of these commutants by explicit computations. Yet, in a more general setting, it would be desirable to establish criteria which guarantee their nontriviality. The results in $[7,10,11,19-23]$, which were also partly used in the present investigation, indicate that relevant information to that effect is contained in the modular structure of the inclusions, although the criterion of modular nuclearity, put forward in [10], seems to be too stringent. It would be desirable to replace it by a less restrictive condition of a similar nature which covers a wider range of examples and can be checked more easily in models. 


\section{Acknowledgements}

DB wishes to thank the Institute for Fundamental Theory and the Department of Mathematics of the University of Florida, and SJS wishes to thank the Institute for Theoretical Physics of the University of Göttingen for hospitality and financial support which facilitated this research. This work was supported in part by a research grant of Deutsche Forschungsgemeinschaft (DFG).

\section{References}

[1] H. Araki, On the algebra of all local observables, Prog. Theor. Phys., 32, 844-854 (1964).

[2] J.J. Bisognano and E.H. Wichmann, On the duality condition for a Hermitian scalar field, J. Math. Phys., 16, 985-1007 (1975).

[3] J.J. Bisognano and E.H. Wichmann, On the duality condition for quantum fields, J. Math. Phys., 17, 303-321 (1976).

[4] H.-J. Borchers, On revolutionizing quantum field theory with Tomita's modular theory, J. Math. Phys., 41, 3604-3673 (2000).

[5] H.-J. Borchers, D. Buchholz and B. Schroer, Polarization-free generators and the S-matrix, Commun. Math. Phys., 219, 125-140 (2001).

[6] O. Bratteli and D.W. Robinson, Operator Algebras and Quantum Statistical Mechanics I, Berlin, Heidelberg, New York: Springer-Verlag, 1979.

[7] R. Brunetti, D. Guido and R. Longo, Modular localization and Wigner particles, Rev. Math. Phys., 14, 759-785 (2002).

[8] D. Buchholz, S. Doplicher and R. Longo, On Noether's theorem in quantum field theory, Ann. Phys., 170, 1-17 (1986).

[9] D. Buchholz, O. Dreyer, M. Florig and S.J. Summers, Geometric modular action and spacetime symmetry groups, Rev. Math. Phys., 12, 475-560 (2000).

[10] D. Buchholz and G. Lechner, Modular nuclearity and localization, Ann. Henri Poincaré, 5, 1065-1080 (2004).

[11] D. Buchholz and S.J. Summers, Stable quantum systems in Anti-de Sitter space: Causality, independence and spectral properties, J. Math. Phys., 45, 4810-4831 (2004).

[12] D. Buchholz and S.J. Summers, Quantum statistics and locality, Phys. Lett. A, 337, 17-21 (2005).

[13] S. Doplicher, K. Fredenhagen and J.E. Roberts, The quantum structure of spacetime at the Planck scale and quantum fields, Commun. Math. Phys., 172, 187-220 (1995).

[14] S. Doplicher and R. Longo, Standard and split inclusions of von Neumann algebras, Invent. Math. 75, 493-536 (1984). 
[15] M.R. Douglas and N.A. Nekrasov, Noncommutative field theory, Rev. Mod. Phys., 73, 977-1029 (2001).

[16] D.A. Eliezer and R.P. Woodard, The problem of nonlocality in string theory, Nucl. Phys., B325, 389-469 (1989).

[17] R. Jost, General Theory of Quantized Fields, (American Mathematical Society, Providence, RI), 1965.

[18] R.V. Kadison and J.R. Ringrose, Fundamentals of the Theory of Operator Algebras, Volume II, Orlando: Academic Press, 1986.

[19] G. Lechner, Polarization-free quantum fields and interaction, Lett. Math. Phys., 64, 137-154 (2003).

[20] G. Lechner, On the existence of local observables in theories with a factorizing S-matrix, J. Phys. A, 38, 3045-3056 (2005).

[21] G. Lechner, An existence proof for interacting quantum field theories with a factorizing S-matrix, e-print: arXiv math-ph/0601022

[22] R. Longo and K.-H. Rehren, Local fields in boundary conformal QFT, Rev. Math. Phys., 16, 909-960 (2004).

[23] J. Mund, B. Schroer and J. Yngvason, String-localized fields and modular localization, Commun. Math. Phys., 268, 621-672 (2006).

[24] H. Roos, Independence of local algebras in quantum field theory, Commun. Math. Phys., 16, 238-246 (1970).

[25] S. Schlieder, Einige Bemerkungen über Projektionsoperatoren, Commun. Math. Phys., 13, 216-225 (1969).

[26] B. Schroer, Modular localization and the bootstrap-formfactor program, Nucl. Phys. B, 499, 547-568 (1997).

[27] B. Schroer, Modular wedge localization and the $d=1+1$ formfactor program, Ann. Phys., 275, 190-223 (1999).

[28] R.F. Streater and A.S. Wightman, PCT, Spin and Statistics, and All That (Reading, Mass., Benjamin/Cummings Publ. Co.), 1964. 